\title{
Differential Synthesis of Secondary Metabolites by Streptomyces chrestomyceticus Strain ADP4 in Response to Modulation in Nitrogen Source and its Anti-Candida Activity ${ }^{\dagger}$
}

\author{
Radha Singh and Ashok K. Dubey* \\ 1 Department of Biological Sciences and Engineering, Netaji Subhas University of Technology, Dwarka, \\ New Delhi 110078, dharana.radha@gmail.com; akdubey@nsut.ac.in* \\ * Correspondence: akdubey@nsut.ac.in; Tel.: +91 8920042038 \\ + Presented at the 1st International Electronic Conference on Microbiology, 2-30 November 2020; Available \\ online: https://ecm2020.sciforum.net/
}

Published: 2 November 2020

\begin{abstract}
Streptomyces spp. are known producers of therapeutically important secondary metabolites with diversity in structure and function. Optimization of different bioprocess parameters towards the improved productivity and activity profile is always considered to be of high significance. Peptone is an acclaimed source of nutrients that had been studied for its effect on the bioactivity of metabolites produced during media development. In the present communication, we report remarkable variation in the profile and anti-Candida activity of the metabolites produced by Streptomyces chrestomyceticus strain ADP4 in response to variation in the source of peptone. It was found that peptone procured from different manufactures (Himedia, CDH and Diffco) have shown noteworthy variation when used as a component of Sabouraud Dextrose Broth (SDB). The zone of inhibition, values of minimum inhibitory concentration $\left(\mathrm{MIC}_{90}\right.$ ) against $\mathrm{C}$. krusei and the yield of metabolites varied significantly when the metabolites were produced in SDB medium using peptones from above mentioned sources. $\mathrm{CDH}$-peptone was found to be the best for antiCandida activity with highest zone of inhibition of $38 \pm 2.0 \mathrm{~mm}$ and MIC 90 value of $2.877 \pm 0.22 \mu \mathrm{g} / \mathrm{mL}$ against C. krusei ATCC 6258. Activity against $C$. albicans ATCC 10231, C. tropicalis ATCC 750, C. parapsilosis ATCC 90028 and C. auris CBS 12372 was also better as compared to the activities obtained using other peptones. The total yield of the metabolites was approximately twice in SDB with $\mathrm{CDH}$-peptone as compared to those with peptone from HiMedia and Diffco. Analyses of the metabolites by using TLC and HPLC demonstrated a clear and significant difference in their profile. Since peptone is a major nitrogen source, it may be inferred that nitrogen source may play a critical role in regulation of biosynthetic gene clusters associated with synthesis of anti-Candida compounds by S. chrestomyceticus ADP4.
\end{abstract}

Keywords: Streptomyces spp., anti-Candida; Minimum Inhibitory Concentration; Peptone; Secondary Metabolites

\section{Introduction}

Candida spp. are part of normal human microbiome but are important opportunistic pathogens. They have been reported to cause superficial and systemic infections in immunocompromised and susceptible individuals [1].

The increasing incidences of drug resistance among pathogens is the major threat for the human health, which highlight the need for discovery of new therapeutic agents as potential drug 
candidates. Actinobacteria in general and members of the genus, Streptomyces in particular have been well recognized for their ability to produce a plethora of antibiotics. It has been known that the Streptomyces spp. contribute about $80 \%$ in the production of bioactive compounds out of all actinobacteria [2]. The structural and functional diversity of secondary metabolites make them an inexhaustible resource for the drug discovery [3].

The production of secondary metabolites in the microorganisms is governed by biosynthetic gene clusters whose expression can be modulated by factors like carbon and nitrogen sources and $\mathrm{pH}$ [4]. The composition of complex nitrogen sources like yeast extract and peptone sourced from different manufacturers, might vary in respect of the composition of amino acids and peptides [5]. Bacteriological peptone is an enzymatic digest of animal tissues (meat). It has high content of tryptophan along with the presence of other amino acids that supports the growth of the microorganisms including Streptomyces chrestomyceticus [6]. However, the composition of peptone depends on the source and condition of extraction that may vary from manufacture to manufacture and even in among lots of the same manufacturer [7].

In the present study, the effect of peptone, procured from different manufacturers, on the production antifungal metabolites by $S$. chrestomyceticus ADP4 with activities against $C$. albicans, $C$. krusei, C. tropicalis, C. parapsilosis and C. auris is reported.

\section{Methods}

S. chrestomyceticus strain ADP4, used to produce secondary metabolites, has been described earlier [6]. Culture was maintained at $28^{\circ} \mathrm{C}$ and stored at $4{ }^{\circ} \mathrm{C}$ on Nutrient Agar (pH 7.0). Test pathogens were C. albicans ATCC 10231, C. krusei ATCC 6258, C. tropicalis ATCC 750, C. parapsilosis ATCC 90028 and C. auris CBS 12372, which were maintained at $37{ }^{\circ} \mathrm{C}$ on Sabouraud Dextrose Agar (SDA, pH 5.6).

Single colony of 48 hour grown ADP4 culture was transferred to $3 \mathrm{~mL}$ SDB and incubated at 28 ${ }^{\circ} \mathrm{C}$ and $150 \mathrm{rpm}$ for $72 \mathrm{~h}$. This inoculum was transferred to $300 \mathrm{~mL}$ SDB for production of secondary metabolites. The SDB was prepared with peptone procured from different brands: Himedia (India), Diffco (United states) and CDH (India). Production was carried out for a period of 4-days under the conditions mentioned above. The metabolites were extracted, and their anti-Candia activities were characterized as reported earlier [6]. Metabolite profiling of the extract was done by using reverse phase HPLC on C18 column.

\section{Results and Discussion}

The maximum zone of inhibition was observed against all the test pathogens on day 4 in the culture broth of SDB with Himedia- peptone and Diffco- peptone. But highest activity was observed on day 4 that continues till day 8 . So, all the cultures were harvested on day 4 . The interesting observation was recorded in SDB with $\mathrm{CDH}$-peptone that activity increased drastically against all the test pathogens. However, the activity against C. krusei ATCC 6258 had seen remarkable increase. Also, highest dry cell mass of $205 \mathrm{mg} / \mathrm{L}$ was obtained in SDB with CDH-peptone followed by 87.3 $\mathrm{mg} / \mathrm{L}$ and $48.5 \mathrm{mg} / \mathrm{L}$ in SDB with the peptone from Diffco and Himedia respectively.

The liquid-liquid extraction of all the broths were active against all the mentioned test pathogens. The yield of the metabolite extract was found to be highest in the SDB with CDH-peptone (323 mg/L) as compared to the SDB with Himedia- peptone (202 mg/L) and with Diffco-peptone (185 $\mathrm{mg} / \mathrm{L})$.

As the results from well diffusion assay were semi-qualitative, a more accurate estimation of the potency of the extracts was obtained by determining $\mathrm{MIC}_{90}$ values, which are presented in table 1.

Table 1. MIC90 values of ADP4 metabolite extract against Candida pathogens:.

\begin{tabular}{ccccc}
\hline \multicolumn{5}{c}{ Minimum inhibitory concentration $(\mu \mathrm{g} / \mathrm{mL})$} \\
\hline C. albicans & C. krusei & C. tropicalis & C. parapsilosis & C. auris \\
ATCC 10231 & ATCC 6258 & ATCC 750 & ATCC 90018 & CBS 12372 \\
\hline
\end{tabular}




\begin{tabular}{cccccc}
\hline $\begin{array}{c}\text { SDB with CDH } \\
\text { peptone }\end{array}$ & $0.725 \pm 0.07$ & $3.57 \pm 1.022$ & $6.495 \pm 0.120$ & $5.915 \pm 0.34$ & $7.78 \pm 0.52$ \\
\hline $\begin{array}{c}\text { SDB with } \\
\begin{array}{c}\text { Himedia } \\
\text { peptone }\end{array}\end{array}$ & $30.69 \pm 1.626$ & $55.7 \pm 0.737$ & $38.86 \pm 1.313$ & $61.24 \pm 1.823$ & $\begin{array}{c}31.253 \pm \\
2.129\end{array}$ \\
\hline
\end{tabular}

The data suggests that the SDB with $\mathrm{CDH}$ - peptone is highly potent having very low $\mathrm{MIC}_{90}$ value as compared to the SDB with Himedia- peptone against all the test pathogens. Also, the activity of SDB with CDH-peptone was found to be higher as compared to the recently reported Streptomyces spp. [7, 8].

To look for the reason behind the significant difference in the potency of these extracts, the analytical RP-HPLC was performed for SDB with CDH-peptone and SDB with Himedia-peptone. The chromatogram of RP-HPLC clearly indicates the difference among the metabolite profile of two extracts as shown in figure 1 .

A.

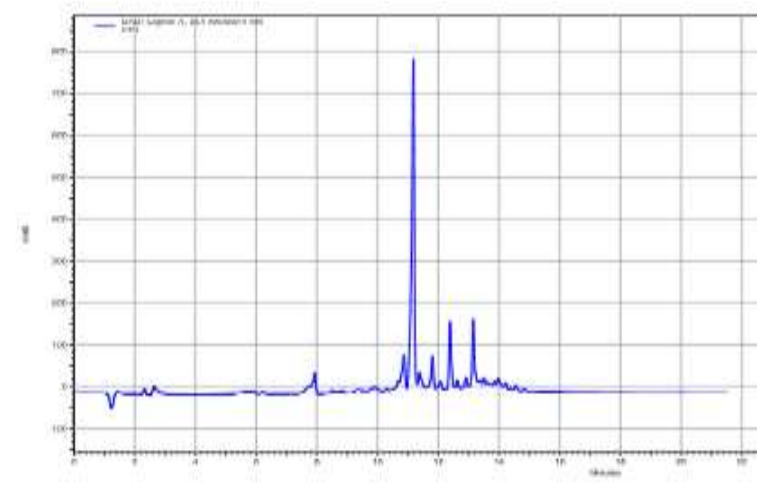

G:V018\ResultiCE 1, rsittCE1. dat, DAD: Signal A, $254 \mathrm{~nm} / \mathrm{Bw}: 4 \mathrm{~nm}$
B.

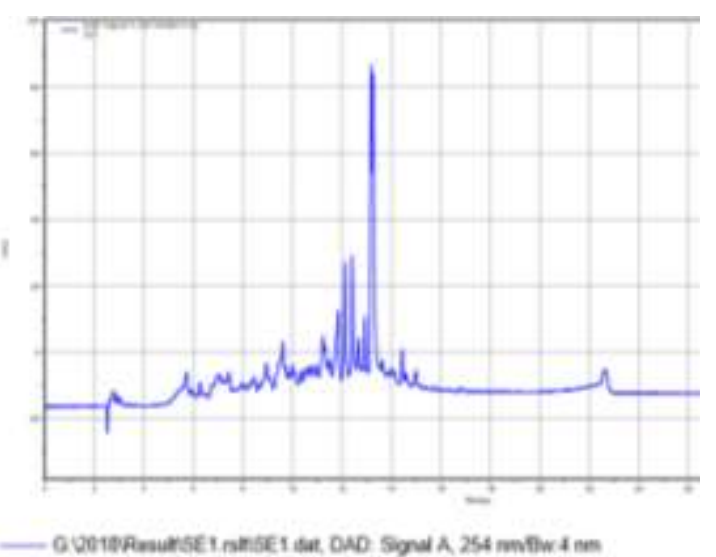

Figure 1. RP- HPLC of Metabolite extracts. A. SDB with CDH- peptone and B. SDB with Himediapeptone.

The results clearly indicated the difference in the metabolite profiles of the extracts that may confer difference in their potency. From overall results of the yield and activity profile, it can be suggested that the same component of culture medium like peptone but from different brands have significant effect on S. chretomyceticus strain ADP4 physiology as well as in the production different of metabolites in the medium that vary in their potency. However more clear reason behind the difference in the metabolite production by strain ADP4 required to be investigated further.

\section{Conclusion}

Peptone procured from different brands had a remarkable effect on the production of several anti-fungal metabolites in S. chretomyceticus strain ADP4. Since peptone primarily served as complex source of nitrogen in the medium, it can be inferred that the expression of biosynthetic gene clusters (BGCs) in S. chrestomyceticus strain ADP4 can be modulated by nitrogen. However, the precise and predictable regulation of BGCs for production of specific or anti-Candida compounds need in-depth investigation.

Author Contributions: A.K.D. has conceptualized the research project and guided the experimental work. R.S. has carried out the experiments and prepared the initial draft of the manuscript, which was corrected and finalized by A.K.D. All authors have read and agreed to the published version of the manuscript.

Funding: “This research was funded by DST-SERB, India, DST-SERB EMR/2017/000254. No funding for APC was provided by any agency.

Acknowledgments: The research grant by the DST - SERB, Government of India to carry out this work is duly acknowledged. 
Conflicts of Interest: “The authors declare no conflict of interest."

\section{References}

1. Naglik, J.R.; König, A.; Hube, B.; Gaffen, S. L. Candida albicans-epithelial interactions and induction of mucosal innate immunity. Curr. Opin. Microbiol. 2017, 40, 104-112. https://doi.org/10.1016/j.mib.2017.10.030.

2. Bérdy, J. Thoughts and facts about antibiotics: where we are now and where we are heading. J. Antibiot. (Tokyo). 2012 65, 385-395. doi: 10.1038/ja.2012.27.

3. Procópio, R.E.; Silva, I. R.; Martins, M.K.; Azevedo, J.L.; Araújo, J.M. Antibiotics produced by Streptomyces. Braz J. Infect. Dis. 2012, 16, 466-471. https://doi.org/10.1016/j.bjid.2012.08.014

4. Brakhage A.A. Regulation of fungal secondary metabolism. Nat. Rev. Microbiol. 2012, 11, 21-32. doi: 10.1038/nrmicro2916.

5. Sørensen, K.; Van den Broucke, S.; Pelikan, J.M.; Fullam, J.; Doyle, G.; Slonska, Z.; Kondilis, B.; Stoffels, V.; Osborne, R. H.; Brand, H. Measuring health literacy in populations: illuminating the design and development process of the European Health Literacy Survey Questionnaire (HLS-EU-Q). BMC Public Health. 2013, 13, 948. https://doi.org/10.1186/1471-2458-13-948.

6. Srivastava, V.; Dubey, A.K. Anti-biofilm activity of the metabolites of Streptomyces chrestomyceticus strain ADP4 against Candida albicans.J. Biosci. Bioeng. 2016, 122, 434-440. https://doi.org/10.1016/j.jbiosc.2016.03.013.

7. Escalante-Réndiz, D.; de-la-Rosa-García, S.; Tapia-Tussell, R.; Martín, J.; Reyes, F.; Vicente F.; GamboaAngulo, M. Molecular Identification of Selected Streptomyces Strains Isolated from Mexican Tropical Soils and their Anti-Candida Activity. Int. J. Environ. Res. Public. Health. 2019 16, 1913. doi: 10.3390/ijerph16111913.

8. Ambarwati, A.; Wahyuono, S.; Moeljopawiro, S.; Yuwono, T. Antimicrobial activity of ethyl acetate extracts of Streptomyces sp. CRB46 and the prediction of their bioactive compounds chemical structure. Biodiversitas. 2019, 20. https://doi.org/10.13057/biodiv/d210763.

Publisher's Note: MDPI stays neutral with regard to jurisdictional claims in published maps and institutional affiliations.

(c) 2020 by the authors. Submitted for possible open access publication under the terms and conditions of the Creative Commons Attribution (CC BY) license (http://creativecommons.org/licenses/by/4.0/). 